Original Paper http://ajol.info/index.php/ijbcs http://indexmedicus.afro.who.int

\title{
Influence des conditions pédo-climatiques sur les composantes du rendement de Jatropha curcas (L) dans les zones Nord de savane et Sud forestière de la Côte d'Ivoire
}

\author{
Noupé Diakaria COULIBALY* ${ }^{*}$ Mongomaké KONE, Tchoa KONE, \\ Tanoh Hilaire KOUAKOU et Yatty Justin KOUADIO
}

Université Nangui Abrogoua, UFR des Sciences de la Nature, Laboratoire de Biologie et Amélioration des Productions Végétales ; 02 BP 801 Abidjan 02, Côte d'Ivoire.

*Auteur correspondant; E-mail: coulnoupe@yahoo.fr

\section{RESUME}

Le pourghère (Jatropha curcas L.) est une plante oléagineuse de la famille des Euphorbiacées. Cette plante qui demeure sauvage, a de multiples usages dont la fabrication de biocarburants. Sa domestication vise à sélectionner les accessions exprimant un haut rendement en graines et donc adaptées à une zone particulière. Des accessions de Jatropha curcas ont ainsi été collectées dans dix localités de la Côte d'Ivoire. Celles-ci ont été cultivées sur les sols de parcelles situées au Sud et au Nord du pays. Le dispositif expérimental est en blocs de Fisher complètement randomisés. Les rendements en fruits secs et en graines des accessions ont été estimés. Les résultats obtenus ont montré qu'au Sud, relativement plus humide et à sol plus riche en matières organiques qu'au Nord, les rendements annuels en fruits ont été meilleurs. L'accession AC5 provenant de Bouaké est la mieux adaptée aux deux sites de culture. Les accessions les plus performantes en termes de rendement en graines dans la zone Nord sont AC1 et AC5. Au Sud, AC3, AC4, AC5 et AC10 se sont révélées les plus intéressantes.

() 2015 International Formulae Group. All rights reserved.

Mots clés : Jatropha curcas, accessions, composantes du rendement, biocarburant, graines, localités de la Côte d'Ivoire.

\section{Influence of soil and climate conditions on the components performance of Jatropha curcas $(L)$ in the northern areas of savannah and forest south of Côte d'Ivoire}

\begin{abstract}
The jatropha (Jatropha curcas L.) is an oleaginous plant of the family Euphorbiaceae. This plant remains wild and has multiple uses including the manufacture of biofuels. Its domestication aims to select the accessions expressing a high seed yield and therefore adapted to a particular area. The Jatropha curcas accessions have been collected in ten towns in the Ivory Coast. These were grown on soils of land located in
\end{abstract}


the South and North. The experimental design is completely randomized block design. The yields of dried fruits and seeds accessions were estimated. The results obtained showed that in the South, with relatively wet soil and richer in organic matter than in the North, fruits in annual returns were better. The AC5 accession from Bouake is best suited to both cultivation sites. The most successful accessions in terms of seed yield in the Northern Zone are AC1 and AC5. In the South, AC3, AC4, AC5 and AC10 have proved the most interesting.

(C) 2015 International Formulae Group. All rights reserved.

Keywords: Jatropha curcas, accessions, yield components, biofuel, seeds, areas of Côte d'Ivoire.

\section{INTRODUCTION}

Jatropha curcas (L.), communément appelé Jatropha, est un arbuste appartenant à la famille des Euphorbiacées. La plante se développe dans de nombreuses régions tropicales et subtropicales (King et al., 2009). Elle est résistante à la sécheresse (Behera et al., 2010 ; Pompelli et al., 2010), et peut croître dans des zones inadaptées à la plupart des cultures (Openshaw, 2000 ; Reubens et al., 2011). Sa tolérance à divers sols et conditions climatiques permet sa large distribution géographique (Jongschaap et al., 2007 ; Babacar et al., 2012).

Dans de nombreux pays, la plante est utilisée par les populations comme haie vive pour protéger les cultures contre les animaux et réduire les dommages ainsi que l'érosion causés par le vent et l'eau. Elle sert aussi de barrière pour séparer les propriétés des enclos pour le cheptel. Traditionnellement, les femmes utilisent les graines de Jatropha pour des traitements médicaux et la production locale de savon. L'huile issue des graines peut servir à la fabrication de biocarburants (Brittaine et al., 2010). De nombreuses plantes non comestibles existent comme sources de biocarburant. Cependant, J. curcas occupe le premier rang de toutes ces cultures en tenant compte des aspects sociaux, économiques (Achten et al., 2010; Behera et al., 2010; Reubens et al., 2011). Les graines de Jatropha contiennent 25 à 32\% d'huile (Pompelli et al., 2010; Misra et Misra, 2010) avec un rendement de 1,5 tonnes d'huile par hectare après cinq années de culture (Tiwari et al., 2007; King et al., 2009).

La production des graines est d'environ 0,8 kilogrammes par mètre de haie par an, avec un rendement en huile de 0,17 litres (Henning, 2003). Une très grande variabilité allant de 0,4 à 12 tonnes/ha/an a été rapportée par Openshaw (2000).

De nombreux rapports sur le rendement de Jatropha sous diverses conditions climatiques à travers le monde restent peu fiables. L'évaluation systématique du rendement en graines des arbres adultes étant d'introduction récente, l'appréciation des valeurs obtenues antérieurement devrait se faire avec circonspection (Achten et al., 2010).

L'un des aspects importants de la domestication de la plante est le degré auquel différents génotypes expriment les meilleures performances sous différentes conditions de culture (Daniel et al., 2005 ; Eriksson et al., 2007 ; Gandonou, 2012). Très peu d'informations sont actuellement disponibles sur la variabilité de production du Jatropha selon la provenance et les conditions écologiques du site de culture. (Atchen et al., 2010). La rareté de données dans ce domaine souligne l'importance d'évaluer le matériel génétique dans divers environnements et de déterminer des zones appropriées de collecte de graines pour l'obtention de matériel génétique de qualité supérieure.

La culture du Jatropha serait une aubaine pour la Côte d'ivoire car l'utilisation 
de son biocarburant pourrait non seulement réduire les émissions de gaz à effet de serre, mais aussi réduire la facture pétrolière. $\mathrm{Sa}$ culture pourrait aussi être une source de revenus additionnels pour la population par la vente des graines.

En effet, les changements climatiques globaux, résultants de la pollution par les gaz à effet de serre tels le dioxyde de carbone et autres constituent une réelle menace pour la sécurité alimentaire dans le tiers monde, voire pour l'humanité entière. Les émissions de gaz à effet de serre ont directement et indirectement des effets négatifs sur la biodiversité. D'après Ouattara et al. (2013), une hausse de la température moyenne annuelle de $0,3{ }^{\circ} \mathrm{C}$ par an, se serait signalée en Côte d'Ivoire depuis la décennie 1990.

Cependant, la culture du Jatropha en Côte d'Ivoire se heurte à quelques contraintes. Entre autres, le manque de promotion par les gouvernants, les populations qui l'utilisent qu'en guise de clôture dans les villages et surtout peu d'instituts de recherche s'investissent dans des travaux pour sa vulgarisation. Et jusqu'à présent, cette plante qui reste sauvage, doit être domestiquée et améliorée pour sa vulgarisation.

Cette étude pourrait contribuer à la domestication et à l'amélioration de la productivité des accessions collectées.

La présente étude vise ainsi à évaluer l'effet des zones agroécologiques assez contrastées de la Côte d'Ivoire sur les rendements en graines des accessions de Jatropha de diverses provenances. Les objectifs spécifiques de ce travail consistent à : déterminer les propriétés physicochimiques des sols des sites d'étude et leur effet sur les rendements, et sélectionner les accessions adaptées à chacune des zones par rapport au rendement. Les résultats attendus de cette étude contribueront à la vulgarisation de la culture de Jatropha en Côte d'Ivoire.

\section{MATERIEL ET METHODES Sites d'étude}

L'étude a été réalisée dans deux (2) zones écologiques différentes de la Côte d'Ivoire (Figure 1) : à Sinématiali au Nord, (latitude: $9^{\circ} 34^{\prime} 0^{\prime \prime} \mathrm{N}$, longitude : $5^{\circ} 27^{\prime} 0^{\prime \prime} \mathrm{W}$ et altitude : 360 mètres) et à Abidjan au sud, (latitude $5^{\circ} 25^{\prime} 08^{\prime \prime} \mathrm{N}$, longitude $4^{\circ} 01^{\prime} 14^{\prime \prime} \mathrm{W}$ et altitude : 105 mètres). Une grande saison de pluie (de juin à septembre) et une longue saison sèche (d'octobre à mai) sont observées à Sinématiali. Les températures moyennes annuelles fluctuent entre 16 et $36{ }^{\circ} \mathrm{C}$ et la pluviométrie moyenne est de l'ordre de 1200 mm/an (CNRA, 2012). Dans la zone d'Abidjan, quatre saisons sont observées: deux saisons humides (avril à mi-juillet : grande saison des pluies ; septembre à novembre : petite saison des pluies) et deux saisons sèches (mi-juillet à septembre : petite saison sèche; décembre à mars: grande saison sèche). Les températures fluctuent entre 21 et $32{ }^{\circ} \mathrm{C}$ et la pluviométrie moyenne est de l'ordre de 1973 mm/an (CNRA, 2012).

\section{Matériel végétal}

Les graines de Jatropha ont été collectées dans dix zones administratives de la Côte d'Ivoire regroupées en cinq régions géographiques (Figure 1). Dans chacune de ces zones, la récolte des graines a été effectuée sur un seul arbre sélectionné sur la base de son âge avancé et le grand nombre de fruits qu'il porte. Le lot de graines de chacun des arbres échantillonnés représente ainsi une accession (Tableau 1).

\section{Méthodes}

Le dispositif expérimental mis en place sur les sites de culture (Nord et Sud de la Côte d'Ivoire) est constitué de blocs de Fisher complètement randomisés avec trois répétitions. Chaque bloc renferme dix parcelles élémentaires, et chaque parcelle 
élémentaire est constituée d'une ligne de dix poquets. Cinq graines ont été semées dans des poquets de $30 \times 45 \mathrm{~cm}$. L'écartement était de $2 \times 2 \mathrm{~m}$ entre les poquets et $2 \times 2 \mathrm{~m}$ entre les lignes. Les semis ont été effectués sur les deux sites en juin 2012 (saison pluvieuse). Trois mois après la levée, le démariage a été effectué de sorte à disposer d'une plante vigoureuse par poquet et de 10 plants par traitement. Aucun apport d'intrants n'a été effectué à l'exception d'une application de Furadan 5G (carbofuran) pour lutter contre les nématodes et les insectes ravageurs. Le sarclage a été fait selon la nécessité

Analyse physico-chimique des sols des sites d'expérimentation

Des prélèvements de sol ont été effectués dans l'horizon 0-45 cm des sites de culture du Sud et du Nord de la Côte d'Ivoire. Ces échantillons de sols ont été analysés au Laboratoire de Pédologie de l'ESA (Yamoussoukro-Côte d'Ivoire). Les différents dosages ont été effectués selon les méthodes d'Anderson et Ingram (1991) et ont concerné : le $\mathrm{pH}$, le carbone total, l'azote total, le phosphore total, le phosphore assimilable, le potassium, les ions et les bases échangeables.

\section{Evaluation des composantes du rendement}

Les composantes du rendement portent sur le poids sec des fruits et des graines par plant et par accession, les rendements en fruits et en graines par plant et par accession et enfin, le ratio (poids graines/poids fruits) des accessions.

Sur chacun des sites expérimentaux, les fruits ont été récoltés par accession (provenances) sur toutes les plantes de chaque parcelle élémentaire. Ces fruits ont été séchés au soleil pendant une semaine pour déterminer le poids des fruits secs produit par plant puis par accession et le poids total des fruits secs. Un échantillon de ces fruits secs a ensuite été transféré à l'étuve à $105{ }^{\circ} \mathrm{C}$ pendant 24 heures pour obtenir un poids sec constant déterminé à partir de trois lots de 100 fruits. Après le décorticage à la main, le poids de 100 graines a été obtenu par pesée. Cette opération permet de déterminer le ratio qui est le poids de graines/poids de fruits. Ce ratio permet d'estimer le poids de coques produits. Cette opération permet d'avoir une connaissance sur le poids graine et coques des différentes provenances. Il permet aussi de prévoir le rendement en graines dans les productions à grande échelle. Le rendement en graines sèches a été estimé en ramenant la production totale de chaque traitement à l'hectare.

\section{Analyses statistiques}

Les données expérimentales évaluées ont été soumises à une analyse de variance (ANOVA) à l'aide du logiciel Statistica 7.1. Les moyennes des mesures ont ensuite été séparées par les tests de comparaisons multiples de Newman-Keuls, au seuil de 5\%.

\section{RESULTATS}

Caractéristiques physico-chimiques des sols des sites expérimentaux

Les valeurs obtenues après le dosage des deux types de sols sont indiquées dans le Tableau 2. Le pH des sols de culture est acide. L'acidité est plus élevée dans le sol du site expérimental localisé au Nord. Les deux sites présentent des rapports $\mathrm{C} / \mathrm{N}$ sensiblement identiques, avec les teneurs en carbone 11 fois supérieures aux taux d'azote. La granulométrie du sol des deux sites de culture diffère principalement par la quantité de limon qui est relativement plus importante sur le site Nord. Les capacités d'échange cationiques d'une part, et les teneurs en ions calcium, magnésium, et potassium d'autre part, sont plus élevées dans le sol du site Nord. Les teneurs en sodium sont sensiblement égales sur les deux sites. Par contre, le sol du site Sud contient plus de phosphore que celui du site Nord. 


\section{Composantes du rendement de Jatropha sur les sites Nord et Sud}

Les poids secs de fruits, total ou produit par plant et les rendements par hectare estimés par provenance sur chacun des sites de culture, sont représentés sur la Figure 2 et le Tableau 3. L'analyse de la Figure 2 et du Tableau 3 montre que quelle que soit la zone de culture, les accessions AC1, AC2, AC7, AC8 et AC9 ont produit le même poids annuel et le même rendement en fruits. Parmi ces accessions, le poids et les rendements les plus élevé ont été obtenus avec $\mathrm{AC} 1$. Avec les accessions $\mathrm{AC} 3, \mathrm{AC} 4, \mathrm{AC} 5, \mathrm{AC} 6$ et $\mathrm{AC} 10$, le poids et les rendements en fruits récoltés dans la zone Sud du pays est significativement plus important que celui de la zone Nord. Dans la zone Sud, les poids et les rendements en fruits sont identiques chez les accessions AC3, $\mathrm{AC} 4, \mathrm{AC} 5$ et $\mathrm{AC} 10$ mais supérieurs à celui de l'accession AC6. Aucune des accessions étudiées n'a exprimé un poids et un rendement en fruits plus élevé dans la zone Nord par rapport à la zone Sud. Les accessions les plus performantes en termes de production de fruits en zone Sud sont: AC1, AC3, AC4, AC5 et AC10. Au Nord, les accessions les plus performantes ont été $\mathrm{AC} 1$ et à un degré moindre, $\mathrm{AC} 4, \mathrm{AC} 5$ et $\mathrm{AC} 10$.

La Figure 3 illustre le poids de 100 graines produites par les différentes accessions sur les sites de culture. Le poids de 100 graines le plus faible a été obtenu avec l'accession AC7 sur le site Nord. L'accession AC3 a présenté le poids de 100 graines le plus élevé lorsqu'elle est cultivée dans la zone Sud. Les accessions AC1 d'une part, et AC9 d'autre part, ont produit sur chacun des sites d'étude, le même poids de 100 graines. Par contre, le poids de 100 graines observé avec les accessions $\mathrm{AC} 2, \mathrm{AC} 3, \mathrm{AC} 4, \mathrm{AC} 7, \mathrm{AC} 8$ et $\mathrm{AC} 10$ a varié et a été plus important sur le site de culture Sud. Parmi ces accessions, AC3 a exprimé la meilleure performance.

L'analyse du Tableau 4 a montré que quelle que soit la zone de culture, le rendement en graines des accessions $\mathrm{AC} 1$, AC2 et AC9 n'a pas varié. Dans la zone Nord, les rendements les plus élevés ont été obtenus avec les accessions AC1 (1,66 t/ha) et AC5 (1,77 t/ha). Aucune différence statistique n'existe entre ces deux rendements. Le rendement le plus faible a été exprimé par AC7 (0,89 t/ha). Au Sud, les rendements en graines les plus importants sont observés avec les accessions AC3, AC4, AC5 et AC10. Ces rendements sont statistiquement identiques et significativement supérieurs à ceux exprimés par les accessions en zone Nord. Les accessions les plus performantes en termes de rendement en graines dans la zone Nord sont $\mathrm{AC} 1$ et AC5. En zone Sud, ce sont AC3, AC4, AC5 et AC10.

Ratio (poids des graines/poids des fruits) des semences récoltées sur les sites

La Figure 4 représente les valeurs expérimentales des ratios graines / fruits sur les deux sites. Au Nord, les résultats du ratio présentent une variabilité entre accessions. Les accessions chez les quelles les valeurs du ratio sont plus élevées au Nord qu'au Sud sont AC5 et AC9. Par contre, chez les autres accessions, AC2, AC3, AC4, AC6, AC8 et $\mathrm{AC10}$, les valeurs du ratio sont plus élevées au Sud qu'au Nord. Au Nord, les valeurs les plus élevées sont obtenues avec les accessions AC3, AC5, AC6 et AC9. Par contre, au Sud, les valeurs les plus élevées sont obtenues avec les accessions AC2, AC3, AC6, AC8 et AC10.

Quelle que soit la zone de culture, le ratio des accessions $\mathrm{AC} 1$ et $\mathrm{AC} 7$ n'a pas varié. 
Tableau 1: Couleurs caractéristiques des gousses et graines des accessions de Jatropha curcas collectées dans différentes localités de la Côte d'Ivoire.

\begin{tabular}{lcccc}
\hline Régions & Localités & $\begin{array}{c}\text { Codes } \\
\text { accessions }\end{array}$ & Couleur des gousses sèches & Couleur des graines \\
\hline \multirow{2}{*}{ Nord } & Sinématiali & AC1 & Marron-clair & Noir-foncé \\
& Ferké & AC2 & Marron-clair & Noir-foncé + rayures \\
\multirow{3}{*}{ Sud } & Abidjan & AC3 & Marron-foncé + points noirs & Noir-foncé \\
& Grand - Lahou & AC4 & Marron & Noir-foncé \\
\multirow{4}{*}{ Centre } & Toumodi & AC5 & Marron+points noirs & Noir +rayure \\
& Bouaké & AC6 & Marron & Noir +rayure \\
& Man & AC7 & Marron+points noirs & Noir-foncé \\
& Duékoué & AC8 & Marron+points noirs & Noir-foncé + rayures \\
Est & Bondoukou & AC9 & Marron-clair & Noir-foncé + rayures \\
& Abengourou & AC10 & Marron-foncé & Noir + rayures \\
\hline
\end{tabular}

Tableau 2: Valeurs des paramètres physico-chimiques des sols des sites de culture de Jatropha curcas.

\begin{tabular}{|c|c|c|}
\hline \multirow[b]{2}{*}{ Paramètres physico-chimiques } & \multicolumn{2}{|c|}{ Sites expérimentaux } \\
\hline & Nord & Sud \\
\hline 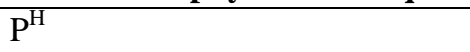 & $5,4 \pm 0,23^{b}$ & $4,1 \pm 0,23^{\mathrm{a}}$ \\
\hline $\mathrm{C}$ & $1,38 \pm 0,06^{\mathrm{a}}$ & $1,5 \pm 0,05^{\mathrm{a}}$ \\
\hline $\mathrm{N}$ & $0,13 \pm 0,01^{\mathrm{a}}$ & $0,12 \pm 0,01^{\mathrm{a}}$ \\
\hline $\mathrm{C} / \mathrm{N}$ & $11,03 \pm 0,23^{\mathrm{a}}$ & $11,41 \pm 0,21^{\mathrm{a}}$ \\
\hline Argile (\%) & $19,23 \pm 0,44^{\mathrm{a}}$ & $23,03 \pm 0,6^{\mathrm{b}}$ \\
\hline Limon fin (\%) & $6,3 \pm 0,17^{\mathrm{b}}$ & $0,78 \pm 0,04^{\mathrm{a}}$ \\
\hline Limon grossier (\%) & $9,13 \pm 0,27^{b}$ & $4,93 \pm 0,82^{\mathrm{a}}$ \\
\hline Sable fin $(\%)$ & $20,19 \pm 0,68^{\mathrm{a}}$ & $20,71 \pm 1,13^{\mathrm{a}}$ \\
\hline Sable grossier $(\%)$ & $44,27 \pm 0,99^{\mathrm{a}}$ & $40,42 \pm 0,84^{\mathrm{a}}$ \\
\hline $\mathrm{CEC}(\mathrm{cmol} / \mathrm{kg})$ & $6,64 \pm 1,15^{\mathrm{a}}$ & $5,52 \pm 0,3^{\mathrm{a}}$ \\
\hline $\mathrm{Ca}^{2+}(\mathrm{cmol} / \mathrm{kg})$ & $1 \pm 0,01^{\mathrm{b}}$ & $0,1 \pm 0,02^{\mathrm{a}}$ \\
\hline $\mathrm{Mg}^{2+}(\mathrm{cmol} / \mathrm{kg})$ & $0,39 \pm 0,03^{\mathrm{b}}$ & $0,03 \pm 0,00^{\mathrm{a}}$ \\
\hline $\mathrm{K}^{+}(\mathrm{cmol} / \mathrm{kg})$ & $0,14 \pm 0,01^{\mathrm{b}}$ & $0,03 \pm 0,01^{\mathrm{a}}$ \\
\hline $\mathrm{Na}^{+}(\mathrm{cmol} / \mathrm{kg})$ & $0,04 \pm 0,01^{\mathrm{a}}$ & $0,05 \pm 0,02^{\mathrm{a}}$ \\
\hline Phosphore total (ppm) & $402 \pm 30,02^{\mathrm{a}}$ & $500 \pm 57,73^{\mathrm{b}}$ \\
\hline Phosphore assimilable (ppm) & $32 \pm 4,61^{\mathrm{a}}$ & $38 \pm 1,73^{b}$ \\
\hline
\end{tabular}


N. D. COULIBALY et al. / Int. J. Biol. Chem. Sci. 9(5): 2346-2361, 2015

Tableau 3: Poids des fruits séchés par plant et rendements en fruits des différentes accessions de Jatropha curcas sur les sites.

\begin{tabular}{|c|c|c|c|c|c|c|c|c|c|c|c|}
\hline Accessions & & $\mathrm{AC} 1$ & $\mathrm{AC} 2$ & AC3 & $\mathrm{AC} 4$ & AC5 & AC6 & $\mathrm{AC7}$ & AC8 & AC9 & AC10 \\
\hline \multirow{2}{*}{$\begin{array}{l}\text { Poids des } \\
\text { fruits/plant (g) }\end{array}$} & Site Nord & $955,2 \pm 64,9^{\mathbf{a}}$ & $699,9 \pm 86^{\mathbf{b}}$ & $564,3 \pm 99,8^{\mathrm{c}}$ & $770,5 \pm 97,8^{d}$ & $818,8 \pm 98,8^{\mathrm{e}}$ & $593,2 \pm 97^{\mathbf{f}}$ & $547,8 \pm 96,6^{\mathbf{c}}$ & $522,2 \pm 58,2^{\mathbf{b}}$ & $6619 \pm 96,5^{\mathbf{b}}$ & $8086 \pm 97,7^{\mathrm{e}}$ \\
\hline & Site Sud & $873,4 \pm 72,2^{\mathrm{a}}$ & $644,5 \pm 56,6^{b}$ & $927,1 \pm 78,9^{a}$ & $943,5 \pm 95,7^{\mathbf{a}}$ & $920,9 \pm 89,6^{\mathbf{a}}$ & $768,7 \pm 77,2^{\mathrm{a}}$ & $556,4 \pm 45,6^{\mathrm{c}}$ & $579 \pm 393^{b}$ & $686,9 \pm 44,9^{b}$ & $903,5 \pm 86,5^{a}$ \\
\hline \multirow{2}{*}{$\begin{array}{l}\text { Rendement en } \\
\text { fruits }(\mathrm{t} / \mathrm{ha})\end{array}$} & Site Nord & $2,38 \pm 0,16^{\mathbf{g}}$ & $1,74 \pm 0,2^{\mathrm{h}}$ & $1,41 \pm 2,31^{\mathbf{h}}$ & $1,92 \pm 0,07^{\text {hg }}$ & $2,04 \pm 0,13^{\mathrm{hg}}$ & $1,48 \pm 0,24^{\mathbf{h}}$ & $1,36 \pm 0,24^{\mathbf{h}}$ & $1,3 \pm 0,14^{\mathbf{h}}$ & $1,65 \pm 0,27^{\mathrm{hg}}$ & $2,02 \pm 0,41^{\mathbf{h}} \mathbf{g}$ \\
\hline & Site Sud & $2,18 \pm 0,18^{\mathrm{g}}$ & $1,61 \pm 0,27^{h}$ & $2,31 \pm 0,03^{\mathrm{g}}$ & $2,35 \pm 0,09^{\mathrm{g}}$ & $2,3 \pm 0,12^{\mathbf{g}}$ & $1,92 \pm 0,07^{\text {hg }}$ & $1,39 \pm 0,02^{\mathrm{h}}$ & $1,44 \pm 0,09^{h}$ & $1,71 \pm 0,11^{\mathrm{hg}}$ & $2,25 \pm 0,35^{\mathrm{g}}$ \\
\hline
\end{tabular}

Dans une même ligne, les valeurs suivies d'une même lettre ne présentent aucune différence significative au seuil de 5\% (test de Newman-Keuls).

Tableau 4: Poids des graines séchés par plant et rendements en graines des différentes accessions de Jatropha curcas sur les sites.

\begin{tabular}{|c|c|c|c|c|c|c|c|c|c|c|c|}
\hline Accessions & & $\mathrm{AC} 1$ & $\mathrm{AC} 2$ & $\mathrm{AC} 3$ & $\mathrm{AC} 4$ & AC5 & AC6 & AC7 & AC8 & AC9 & AC10 \\
\hline \multirow{2}{*}{$\begin{array}{c}\text { Poids des } \\
\text { graines/plant (g) }\end{array}$} & Site Nord & $\begin{array}{c}668,64 \pm 45,48 \\
\text { a }\end{array}$ & $\begin{array}{c}517,92 \pm 63,65 \\
\text { c }\end{array}$ & $\begin{array}{c}445,79 \pm 86,15 \\
\text { b }\end{array}$ & $\begin{array}{c}539,35 \pm 20,74 \\
\text { c }\end{array}$ & $\begin{array}{c}695,98 \pm 47,69 \\
\text { a }\end{array}$ & $468,62 \pm 76,7^{\mathbf{c}}$ & $\begin{array}{c}361,54 \pm 63,77 \\
\text { e }\end{array}$ & $\begin{array}{c}370,76 \pm 41,38 \\
\text { ab }\end{array}$ & $\begin{array}{c}555,99 \pm 93,03 \\
\text { c }\end{array}$ & $\begin{array}{c}493,24 \pm 100,1 \\
\text { c }\end{array}$ \\
\hline & Site Sud & $655,05 \pm 54,2^{\mathrm{a}}$ & $\underset{\text { c }}{573,6 \pm 110,6^{a}}$ & $852,93 \pm 13,5^{\mathbf{f}}$ & $754,8 \pm 30,37^{\mathrm{g}}$ & $\begin{array}{c}755,13 \pm 42,62 \\
\mathrm{~g}\end{array}$ & $\begin{array}{c}668,76 \pm 24,64 \\
\text { a }\end{array}$ & $\begin{array}{c}389,42 \pm 7,19^{\mathbf{a}} \\
\mathbf{b}\end{array}$ & $\begin{array}{c}492,15 \pm 33,43 \\
\mathbf{c}\end{array}$ & $\begin{array}{c}542,65 \pm 35,49 \\
\mathbf{c}\end{array}$ & $\begin{array}{c}804,11 \pm 127,6 \\
\mathbf{h}\end{array}$ \\
\hline \multirow{2}{*}{$\begin{array}{l}\text { Rendement en } \\
\text { graines (t/ha) }\end{array}$} & Site Nord & $1,66 \pm 0,11^{\mathrm{i}}$ & $1,28 \pm 0,15^{\mathbf{j}}$ & $1,11 \pm 0,21^{1}$ & $1,34 \pm 0,05^{\mathbf{j}}$ & $1,73 \pm 0,12^{\mathrm{i}}$ & $1,16 \pm 0,19^{j}$ & $0,89 \pm 0,15^{\mathbf{m}}$ & $0,92 \pm 0,10^{\mathbf{f}}$ & $1,38 \pm 0,23^{\mathbf{j}}$ & $1,23 \pm 0,24^{\mathbf{j}}$ \\
\hline & Site Sud & $1,63 \pm 0,13^{i}$ & $1,43 \pm 0,24 \mathrm{i}^{\mathrm{j}}$ & $2,12 \pm 0,03^{\mathrm{d}}$ & $1,88 \pm 0,07^{\mathrm{d}}$ & $1,88 \pm 0,10^{\mathrm{d}}$ & $1,67 \pm 0,06^{i}$ & $0,97 \pm 0,01^{\mathrm{r}}$ & $1,22 \pm 0,08^{1}$ & $1,35 \pm 0,08^{\mathrm{j}}$ & $2,00 \pm 0,31^{\mathrm{d}}$ \\
\hline
\end{tabular}

Dans une même ligne ou dans une même colonne, les valeurs suivies d'une même lettre ne présentent aucune différence significative au seuil de 5\% (test de Newman-Keuls). 


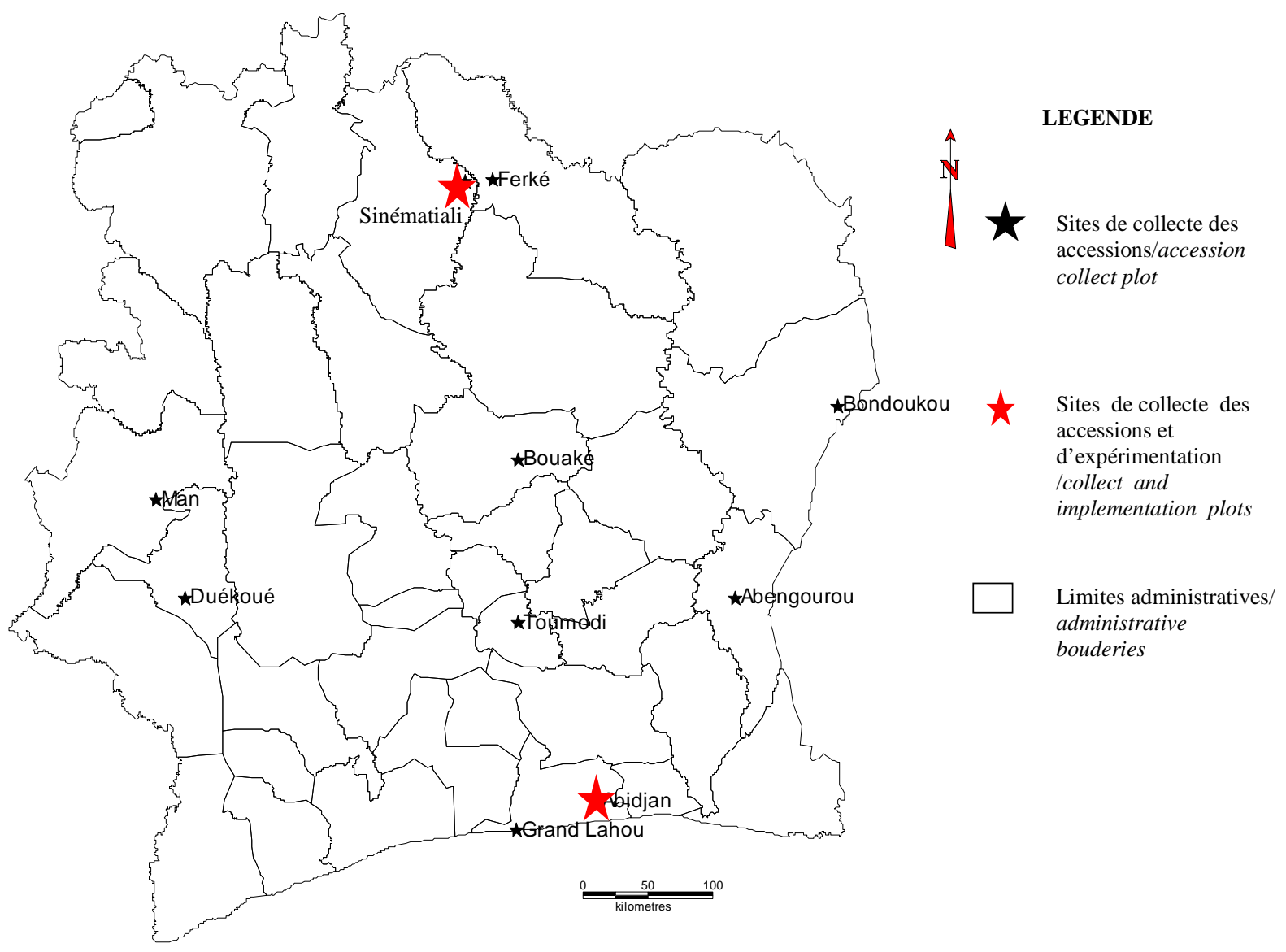

Figure 1: Cartographie des sites de collecte et d'expérimentation de Jatropha curcas en Côte d'Ivoire. 


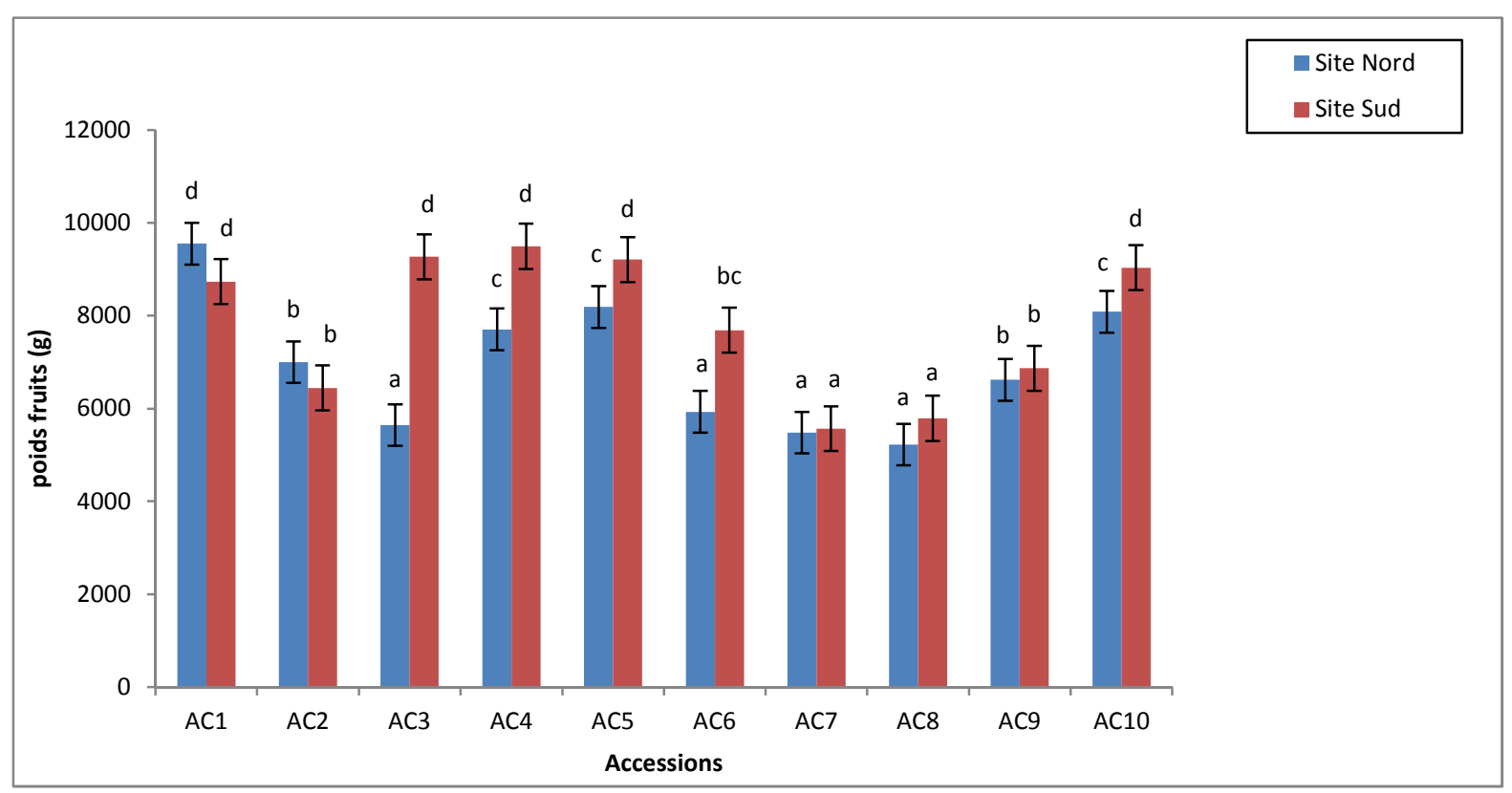

Figure 2: Poids sec annuel des fruits de Jatropha curcas par accession et par site. Les valeurs suivies d'une même lettre ne présentent aucune différence significative au seuil de 5\% (test de Newman-Keuls).

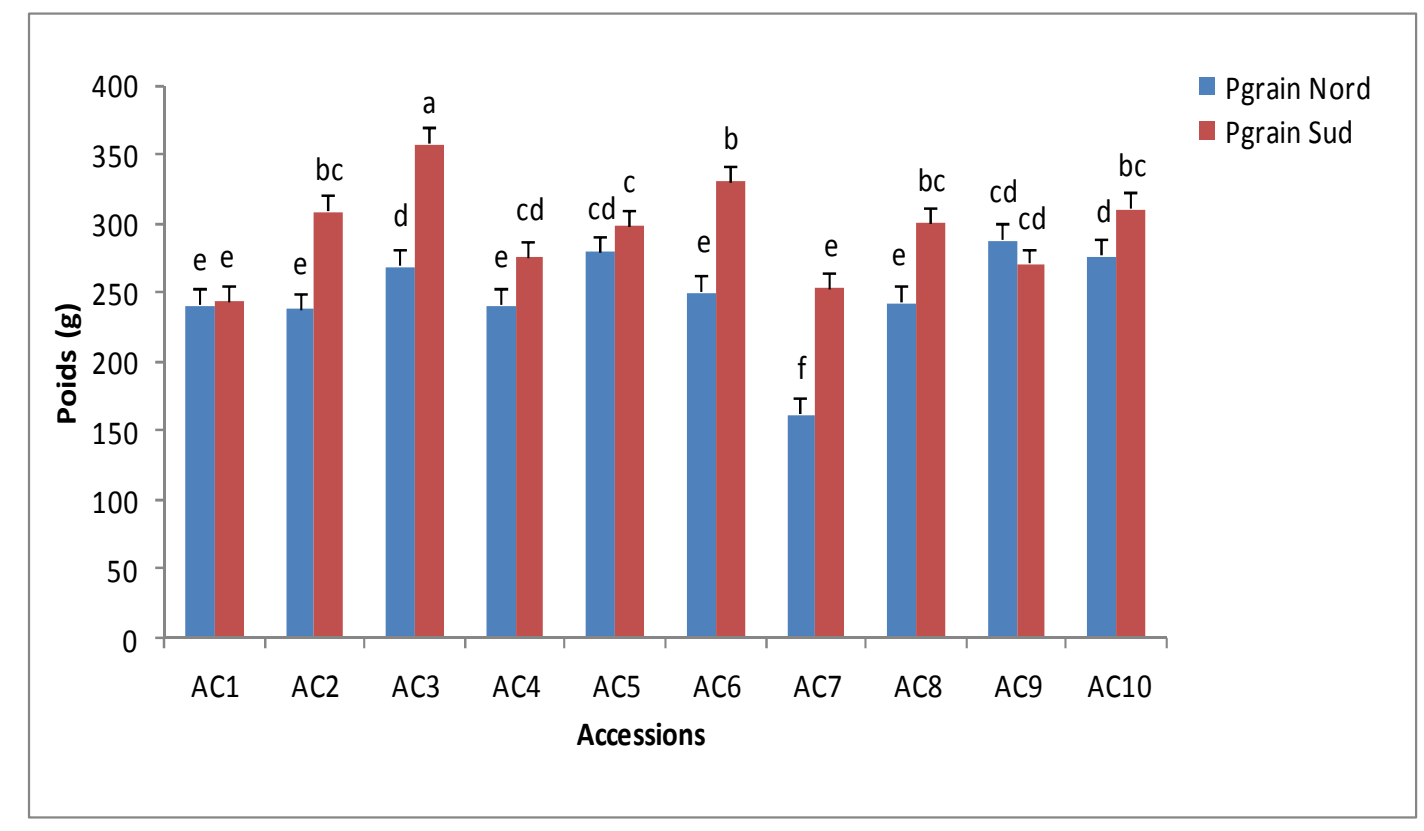

Figure 3: Poids des graines issues de cent fruits de Jatropha curcas récoltés sur les sites Nord et Sud.Les valeurs suivies d'une même lettre ne présentent aucune différence significative au seuil de 5\% (test de NewmanKeuls) ; Pgrain Nord: Poids des graines du site Nord ; Pgrain Sud: Poids des graines du site Sud. 


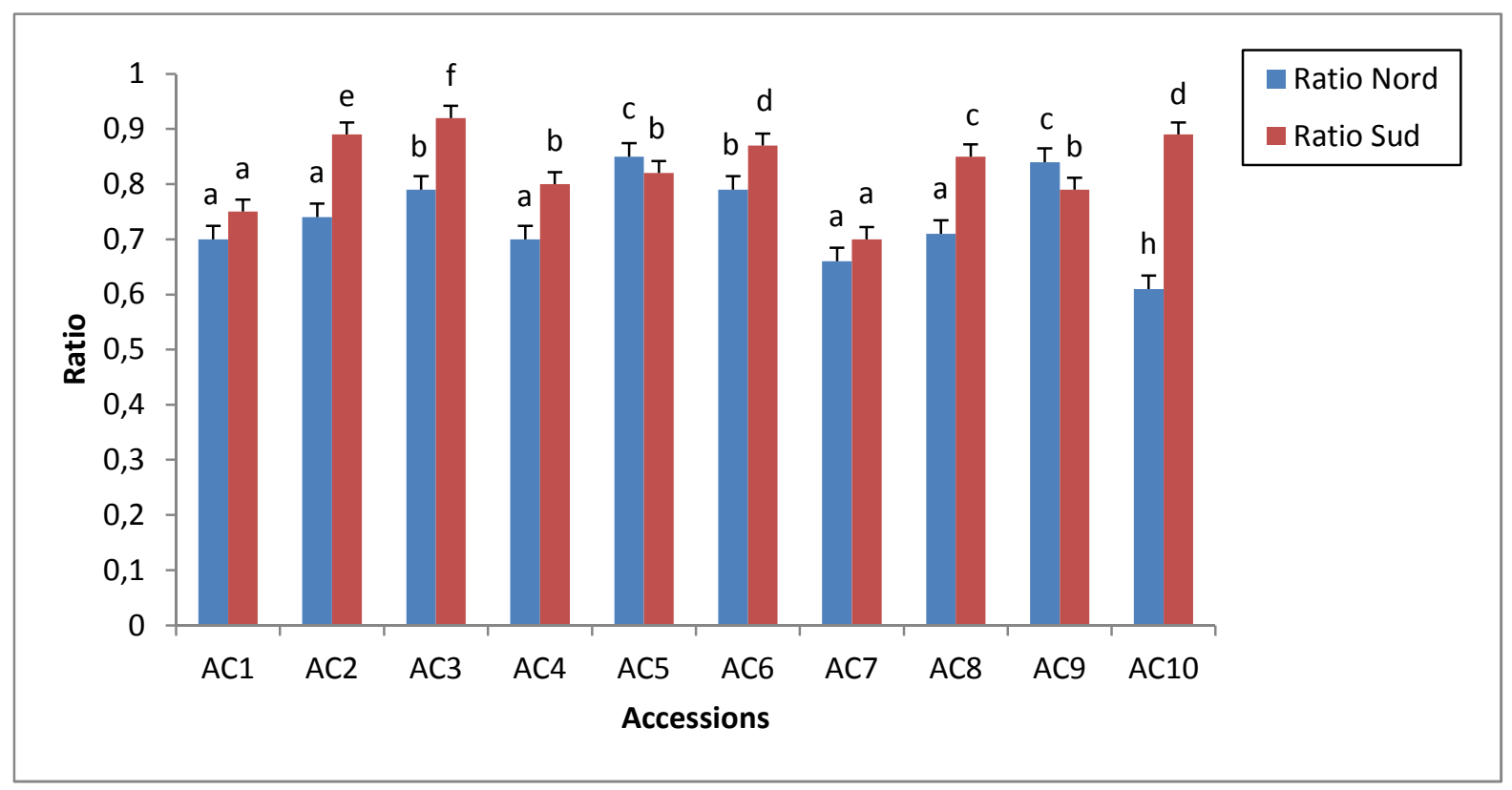

Figure 4: Ratio graines/fruits des sites d'expérimentation Nord et Sud. Les valeurs suivies d'une même lettre ne présentent aucune différence significative au seuil de 5\% (test de Newman-Keuls).

\section{DISCUSSION}

Le nombre de capsules et de graines par plante et les rendements en fruits et en graines ont été plus importants sur le site Sud que celui du Nord. Ces observations témoignent de l'effet du site sur les composantes du rendement. La productivité des accessions a donc été élevée sur le site Sud, malgré la diversité de provenance des accessions. Cette forte production pourrait s'expliquer par le fait de la composition chimique du sol et de la bonne répartition des pluies sur ce site. Le site Sud présente deux saisons pluvieuses contre une au Nord.

Les analyses des éléments chimiques des échantillons de sols des deux sites de culture ont montré que le potassium et le magnésium ont été les éléments majeurs ayant une teneur élevée sur le site Nord. Alors que sur le site Sud, l'élément majeur a été le phosphore assimilable. L'analyse a aussi montré que les sols sont plus acides au Sud qu'au Nord. Il semble que cette acidité élevée au Sud, soit à l'origine de la faible teneur en bases échangeables par rapport au Nord.

Le potassium contribue à l'initiation des boutons floraux et au développement des fruits et des graines; tandis que le magnésium est un élément de la chlorophylle qui contribue à la maturité des fruits et favorise l'absorption du phosphore, de l'azote et du soufre par la plante (Jun et al., 2012). Les différences de rendement entre le site du Nord et celui du Sud peuvent s'expliquer par la différence des teneurs de ces éléments chimiques. Ces résultats sont en accord avec ceux de Reinhardt (2008) qui a défini les besoins théoriques de Jatropha curcas. Selon lui, les éléments clés dans la fertilisation de Jatropha sont l'Azote (N), le Phosphore (P) et le Potassium (K). Il a montré que les meilleurs rendements chez cette espèce peuvent être obtenus sur un site ayant des teneurs en azote de $0,19 \%$, en phosphore de $29,67 \mathrm{mg} / \mathrm{kg}$ et en potassium de $0,11 \mathrm{cmol} / \mathrm{kg}$.

Les rendements en graines des accessions $\mathrm{AC} 1$ et $\mathrm{AC} 9$ ont été identiques sur 
les sites Nord et Sud. Ces accessions semblent moins exigeantes en éléments nutritifs. Chez les accessions AC2, AC3, AC4, AC5, AC6, $\mathrm{AC} 7$ et $\mathrm{AC} 10$, les rendements en graines ont été plus élevés sur le site Sud. Ces accessions semblent exigeantes en phosphore car sur le site Nord, les teneurs en phosphore sont en deçà de la valeur théorique. Par contre, elles ont été moins exigeantes en potassium. Quant à l'accession $\mathrm{AC} 8$, les rendements en graines ont été plus élevés au Nord qu'au Sud. Au vu de ces résultats on pourrait penser que cette accession a été plus exigeante en potassium et moins exigeante en phosphore et en azote. Car les teneurs de ces deux éléments chimiques sont en deçà des teneurs théoriques avancées par Reinhardt (2008).

L'effet des facteurs climatiques des sites de culture sur les composantes du rendement a aussi montré que le poids sec des fruits par plante, le rendement en fruits et le poids de graines par plante ont statistiquement été différents sur les deux sites chez la plupart des accessions. Ceci pourrait montrer qu'il existe une interaction importante entre les provenances et l'environnement. Sur les sites, la pluviométrie a influencé la productivité de toutes les accessions évaluées. Les rendements ont varié d'un site à un autre chez la plupart des accessions. Une étude réalisée en Malaisie sur des populations de $J$. curcas provenant de Malaisie, d'Inde, d'Indonésie et des Philippines (Biabani et al., 2012) indique des différences significatives entre ces populations en ce qui concerne la précocité de floraison, le nombre d'inflorescences, le nombre de fruits par plante, le nombre de graines par plante, le rendement en graines et en huile par hectare lorsqu'elles ont été cultivé sur des sites différents. $\mathrm{La}$ différence entre les pluviométries sur les sites Nord et Sud de la Côte d'Ivoire, durant la période d'étude pourrait être la cause de l'augmentation du rendement sur le site Sud. En effet, la pluviométrie moyenne annuelle enregistrée sur le site Sud a été de $1975 \mathrm{~mm}$ alors que celle obtenue sur le site Nord a été de 1200 mm (CNRA, 2012). Selon Jongschaap et al. (2007), la production de graines de J. curcas est rentable dans des conditions d'humidité variant de 600 à plus de $1000 \mathrm{~mm}$ de pluie par an; l'optimum de production est atteint à $1500 \mathrm{~mm}$.

Relativement aux conditions écologiques, les sites étudiés peuvent être considérés comme propices à la culture du Jatropha ; le Sud étant au-dessus de l'optimum avec $475 \mathrm{~mm}$ et le Nord en dessous avec 300 $\mathrm{mm}$. Les températures moyennes, et surtout les pluviométries relevées annuellement, peuvent permettre d'obtenir une bonne production, quoique pour le dernier facteur cité, les valeurs (1200 mm au Nord et $1973 \mathrm{au}$ Sud) ne sont pas optimales. Il semble ainsi, qu'une bonne productivité du Jatropha tienne compte non seulement de conditions climatiques acceptables, mais également d'un sol profond à texture sablo-argileuse et drainant (Gour, 2006). On peut estimer que les pluies ont été mieux reparties au Sud où deux saisons de pluies sont observées par rapport au Nord qui enregistre une saison de pluies. Les rendements sont globalement élevés au Sud qu'au Nord. Cela pourrait nous amener à penser que la bonne répartition des pluies soit à l'origine de cette différence.

Henning (2003) a également montré que la productivité de l'arbre est dépendante du régime pluviométrique qui détermine le nombre de fructifications et donc de récoltes. D'après Domergue et Pirot (2008), Ouattara et al. (2011) et Wen et al. (2012), on ne peut pas parler de rendement indépendamment des conditions pédoclimatiques du milieu. Selon eux, il serait plus juste de parler de rendement potentiel dans une zone pédoclimatique donnée. La présente étude a été réalisée avec des semences récoltées sur des arbustes subspontanés. Elles n'ont donc fait l'objet d'aucune sélection préalable pour leur productivité dans des zones géographiques ne présentant pas les conditions environnementales du site de mise en culture. 
Cela pourrait expliquer en grande partie les faibles performances de certaines accessions et la très grande variabilité des rendements obtenus d'un site à un autre.

Les résultats montrent l'importance des accessions et de l'environnement dans l'obtention d'une meilleure performance agronomique. Ainsi, la comparaison des accessions a été effectuée afin de détecter les accessions les plus performantes dans leur environnement. Selon Ashraf et al. (2001), Ahoton et al. (2011) et Assogbadjo et al. (2009), l'adaptabilité des variétés à divers environnements est donnée par le degré de son interaction avec différents environnements sous lesquels il est cultivé. Une variété ou un génotype est considéré comme stable si son rendement moyen est élevé et fluctue faiblement lorsque ces variétés sont cultivées sous différents milieux.

La comparaison des accessions a montré que sur le site Nord, les rendements en graines ont varié de 0,61 à 1,11 t/ha et sur celui du Sud, ils ont varié de 0,62 à 1,32 t/ha. Ces rendements obtenus sur les sites sont comparables aux valeurs références de l'ICRISAT (2009) qui indiquent 1,3 t de graines par hectare. Toutefois, de nombreux rapports ont signalé des rendements de 3 à 5 t/ha/an (Ogunwole et al., 2007). Le jeune âge des plants et surtout les conditions pluviométriques pourraient expliquer le faible rendement observé. En effet, les plantes sont âgées de deux ans. Une stabilité du rendement s'observerait à partir de la 7ème année de culture (Gokhale, 2008). Quant aux conditions pluviométriques, Orwa et al. (2009), Jongschaap et al. (2007), Trabucco et al. (2010) et Rajaona et al. (2011) ont affirmé que les précipitations annuelles de $300 \mathrm{~mm}$ permettent d'assurer la survie de la plante et un minimum de $500 \mathrm{~mm}$ par an est nécessaire pour la production des graines. À partir de ce seuil, les rendements augmentent très fortement avec la pluviosité annuelle totale pour atteindre un optimum à $1500 \mathrm{~mm}$ avant de diminuer lentement à mesure que les précipitations annuelles augmentent.

Les rendements obtenus avec les 10 accessions ont été supérieurs à ceux obtenus dans de nombreux pays de l'Afrique. Les rendements obtenus dans ces pays ont varié de 20,40 à 346,2 kg/ha au Congo-Kinshasa (Minengu, 2014) pour une plantation de trois ans avec une pluviométrie moyenne annuelle de $1500 \mathrm{~mm}$, de $123 \mathrm{~kg} /$ ha la deuxième année à $350 \mathrm{~kg} / \mathrm{ha}$ la troisième année avec une pluviométrie de $400 \mathrm{~mm} / \mathrm{an}$ au Sénégal (Diedhiou, 2009) et de 1,2 à 1,5 t/ha pour une plantation âgée de cinq ans sous une pluviométrie de $700 \mathrm{~mm} / \mathrm{an}$ au Burkina Faso (Janin et Ouédraogo, 2009). Les rendements cités dans la littérature diffèrent d'un pays à un autre. Il existe aussi des différences entre les rendements au sein d'un même pays. Toutes ces différences peuvent s'expliquer par les conditions climatiques mais aussi par la variabilité génotypique qui peut exister entre les accessions. Les différences entre ces résultats pourraient non seulement se justifier par les conditions édaphiques et climatiques des zones d'étude, mais aussi par le nombre d'accessions caractérisées par chaque auteur.

Sur le site Nord, le poids des 100 fruits le plus bas et le plus haut sont obtenus respectivement par les accessions AC7 et AC3. De plus, le rapport graine-fruit montre une disproportionnalité entre le poids de fruits et de graines. En effet, les rapports les plus élevés n'ont pas été obtenus avec les poids des fruits les plus élevés. En revanche, AC5 dont le poids de fruits était plus bas par rapport à celui de $\mathrm{AC} 1$, a obtenu un poids de graines supérieur à celui de $\mathrm{AC} 1$. Cela signifie que les graines de cette accession pèsent plus que celles de $\mathrm{AC} 1$.

Sur le site Sud, la tendance est la même que celle décrite sur le site Nord. AC1 a enregistré un poids de fruits plus important que celui de AC6. Mais AC6 dont le poids de fruit est le plus faible a un poids de graines statistiquement identique à celui de $\mathrm{AC} 1$; avec une valeur élevée chez AC6. 
Sur les deux sites, $95 \%$ des accessions ont un ratio supérieur à $65 \%$ et $5 \%$ ont le leur inférieur à $65 \%$. Par contre, les résultats obtenus par Sanou (2010), ont montré que $70 \%$ des provenances ont un rapport poids graine/poids fruit supérieur à $65 \%$. Seulement, $30 \%$ des provenances des accessions qu'il a étudiées, ont un rapport inférieur à $65 \%$. Les rapports les plus bas peuvent être dus à un manque de certains éléments minéraux indispensables pendant le remplissage des fruits c'est-à-dire pendant la formation des graines. Les conditions pédoclimatiques, notamment la pluviométrie pourrait être le facteur primordial qui influence le rendement en fruits et surtout en graines des plantes de Jatropha curcas.

En effet, Diedhiou (2009) a obtenu sous une pluviométrie, moyenne annuelle de $400 \mathrm{~mm}$, un rapport de l'ordre de 0,53. Alors que Sanou (2010) a établi un rapport de l'ordre de 0,63 à 0,68 avec une pluviométrie annuelle de $900 \mathrm{~mm}$. En Côte d'Ivoire, Sur le site Nord, la pluviométrie moyenne annuelle a été de $1200 \mathrm{~mm}$ et un ratio de 0,61 à $0,85 \mathrm{a}$ été obtenu. Sur le site Sud, la pluviométrie moyenne annuelle a été de $1800 \mathrm{~mm}$. Un ratio variant de 0,7 à 0,92 y a été enregistré.

Dans le cadre de cette étude, sur le site Nord, les plus hauts rendements ont été obtenus par les accessions AC1 et AC5. Par contre, les rendements les plus faibles ont été observés chez l'accession AC7.

Sur le site Sud, les rendements les plus élevés ont été obtenus avec les accessions $\mathrm{AC} 3, \mathrm{AC} 4, \mathrm{AC} 5$ et AC10. L'accession AC7 a enregistré le rendement le plus faible.

Les accessions chez lesquels le rendement a moins fluctué dans les conditions agroécologiques du Nord et du Sud, sont les accessions AC1 (1,66 t/ha sur le site Nord et 1,63 t/ha sur le site Sud) et AC9 (1,38 t/ha sur le site Nord et 1,35 t/ha sur le site Sud). Parmi ces accessions, la plus performante et celle qu'on peut introduire dans un programme de recherche est AC5.

\section{Conclusion}

Les caractéristiques physico-chimiques du sol des sites d'études ont été déterminées à l'issue des travaux. L'étude a montré que les caractéristiques du sol et du climat ont influencé les composantes du rendement des accessions du Jatropha. Une différence significative a été observée entre les rendements des accessions utilisées. Les rendements en fruits dépendent de la capacité intrinsèque de chaque accession. Les accessions, AC1 provenant de Sinématiali et AC5 provenant de Bouaké, semblent les plus adaptées et les plus productives de toutes les accessions Les rendements ont été globalement élevés au Sud qu'au Nord. Les conditions pédoclimatiques semblent être le facteur le plus déterminant vis-à-vis de la productivité en fruits sur le site Sud que celui $\mathrm{du}$ Nord. Cette étude nécessite d'autres recherches complémentaires. Notamment la poursuite des tests de rendement sur plusieurs années afin de mieux discriminer les accessions les plus productives, l'extraction des huiles des différentes accessions afin de déterminer l'influence des conditions pédoclimatiques sur les rendements et la qualité des huiles.

\section{CONFLIT D'INTERET}

Les auteurs ne déclarent aucun conflit d'intérêt.

\section{CONTRIBUTIONS DES AUTEURS}

NDC était l'investigateur principal; KM a contribué à l'analyse statistique et à l'amélioration du manuscrit; TK, THK et YJK ont contribué à la correction et à l'amélioration du manuscrit.

\section{REMERCIEMENTS}

Nos remerciements à l'endroit des autorités de l'Université NANGUI ABROGOUA et à la famille COULIBALY à Sinématiali pour avoir mis à notre disposition des parcelles pour la mise en place des cultures. Nos remerciements également à tous 
nos correspondants dans les lieux de collecte des capsules de Jatropha curcas.

\section{RÉFÉRENCES}

Achten WMJ, Nielsen LR, Aerts R, Lengkeek AG, Kjaer ED, Trabucco A, Hansen JK, Maes WH, Graudal L, Akinnifesi FK, Muys B. 2010. Towards domestication of Jatropha curcas. Futura Science, 1(1): 91-107. DOI 104155/bfs 09.4

Ahoton LEF, Quenum G, Mergeai. 2011. Evaluation agromorphologique et sélection des meilleures accessions de Pourghère (Jatropha curcas L.) introduites au Bénin. Int. J. Biol. Chem. Sci., 5(4): 1619-1627. DOI: http://dx.doi.org/10.4314/ijbcs.v5i4.25

Anderson JM, Ingram JS. 1991. Tropical soil Biology and Fertility: Handbook of Methods. (1ere edn). CAB International: Wallinford, UK. 14 pages

Ashraf M, Qureshi AG, Khan NA, 2001. Genotype-environment interaction in wheat. Online Journal of Biological Sciences, 1(5): 356 - 357. DOI: http://www.researchgate.net/publicatio $\mathrm{n} / 26470084$

Assogbadjo AE, G Amadji R, Glèlè Kakaï A, Mama B, Sinsin P, Van Damme. 2009. Evaluation écologique et ethnobotanique de Jatropha curcas L. au Bénin. Int. J. Biol. Chem. Sci., 3(5): 1065-1077. DOI: 10.4314/ijbcs.v3i5. 51085

Babacar D, Diatta S, Agbangba CE, Barro L, Samba SAN'D, Akpo LE. 2012. Seedsmorpho-Metric Characteristics And Germination Capacity Of Some Jatropha Curcas L. provenances. American-Eurasian Journal of Sustainable Agriculture, 6(4): 215-221. DOI:

http://dx.doi.org/10.4314/ijbcs.v6i2.12

Behera SK, Srivastava P, Tripathi R, Singh JP, Singh N. 2010. Evaluation of plant performance of Jatropha curcas L. under different agro-practices for optimizing biomass - A case study. Biomass Bioenerg, 34: 30-41.

Biabani A, Rafii MY, Saleh GB, Shabanimofrad M, Latif Md A. 2012. Phenotypic and genetic variation of Jatropha curcas L. populations from different countries. Maydica Electronic Publication, 57: 164-174.

Brittaine R, Lutaladio N. 2010. Jatropha: a Smallholder Bioenergy Crop. The Potential for Pro-Poor Development. FAO: Roma, Integrated Crop Management. http://www.fao.org/ docrep/012/i1219e/i1219e.pdf, (06/05/2012).

CNRA (Centre National de Recherche Agronomique). 2012. Bulletin agro météorologique annuel de la Côte d'Ivoire, CNRA, 6 p.

Daniel JN. 2005. Jatropha production options under varied resource conditions. In National Seminar on Biodiesel, 23-24 September 2005. Chennai, India.

Diédhiou. 2009. Impacts potentiels de l'introduction de Jatropha curcas L. dans un contexte de variabilité et changement climatiques : impacts agricoles et environnementaux, intérêts économiques pour les ménages et communautés rurales. Atelier de clôture Cotonou, Bénin, 18-21 octobre. 19 pages.

Domergue M, Pirot R. 2008. Jatropha curcas L. - Rapport de synthèse bibliographique CIRAD, UPR AgroGeneration - Biomasse Energie Systèmes de Culture Annuels. Montpellier, Paris. France. 118 p.

Eriksson G, Ekberg I, Clapham D, 2007. An Introduction to Forest Genetics. SLU, Department of Plant Biology and Forest Genetics: Uppsala, Sweden.

Gandonou Ch. B, Houmba NR, Ahoton LE, Desquilbet S, Fakambi6 K, Datinon B, Marshall E. 2012. Evaluation de la levée et de la croissance chez douze 
accessions de Pourghère (Jatropha curcas) au Bénin. Bulletin de la Recherche Agronomique du Bénin (BRAB). http://www.slire.net. 7 pages

Gokhale D. 2008. Jatropha: Experience of Agro-Forestry \& Wasteland Development Foundation, Nashik, India. International Consultation on Pro-poor Jatropha Development. Syngenta International: Rome.

Gour V, Singh B, Swaminathan R, Ponraj V. 2006. Production practices including post-harvest management of Jatropha curcas. In Proceedings of the biodiesel conference toward energy independance - Focus of Jatropha, Hyderabad, India, New Delhi.

Henning RK. 2003. The Jatropha Booklet: A guide to Jatropha Promotion in Africa. Bagani GbR. Weissensberg, Germany. p5-33. Accessed at http://www.jatropha.de/documents/jcl_ booklet_Africa.pdf 13/8/2009

ICRISAT (International Crops Research Institute for the Semi-Arid Tropics), 2009. Protéger la biodiversité, fournir des options, publication officielle de l'ICRISAT, 35p.

Janin, Ouédraogo,. 2009. Enjeux des agrocarburants au Burkina Faso: cas du Jatropha curcas L. 12p

Jongschaap, Ree, Corré WJ, Bindraban PS, Brandenburg WA, 2007. Claims and Fact on Jatropha curcas L. Global Jatropha curcas evaluation, breeding and propagation. Plant Research International Wageningen, report 158, Wageningen, The Netherlands.

Jun Wang, Wen-Zhao Liu, Ting-Hui Dang, Upendra M. Sainju, 2012, Nitrogen Fertilization Effect on Soil Water and Wheat Yield in the Chinese Loess Plateau. Agronomy Journal 04/2012; 105(1): 143-149. DOI: $10.2134 /$ agronj2012.0067

King AJ, He W, Cuevas JA, Freudenberger, M, Ramiaramanana D, Graham IA.
2009. Potential of Jatropha curcas as source of renewable oil and animal feed. J. Exp. Environ. Bot., 60(10) : 2897-2905. DOI : 10.1093/jxb/erp025

Minengu Mayulu, 2014. Etude des possibilités de culture de Jatropha curcas L. dans la région de Kinshasa en République Démocratique du Congo (RDC). Thèse de Doctorat. Université de LiègeGembloux Agro-bio Tech (Belgique) $178 \mathrm{p}$.

Misra M, Misra A, 2010. Jatropha: The Biodiesel Plant Biology, Tissue Culture and Genetic Transformation - A Review. Int. J. Pure Appl. Sci. Technol. 1(1): 11-24. DOI: www.ijopaasat.in

Ogunwole JO, Patolia JS, Chaudahary DR, Arup Gosh, Chikara J. 2007. Improvment of the quality of a degraded Entisol with Jatropha urcas L. under an Indian Semi-Arid Conditions. In Jatropha curcas L - Rapport de synthèse bibliographique CIRAD, AGROgeneration 116 Expert seminar on Jatropha curcas L. Agronomy and genetics, 26-28 march 2007. 2007. Wageningen, The Netherlands: Fact Foundation.

Openshaw K, A review of Jatropha curcas: an oil plant of unfulfilled promise. Alternative Energy Development Inc, 2000.

Ouattara A, Dayoro K, Fokou G. 2013. Perception des effets sanitaires du changement climatique par les populations urbaines défavorisées de Côte d'Ivoire. Conférence internationale Africa Écosanté 2013, Abidjan, 15 p.

Ouattara B, Ndir KN, Diedhiou I, Diouf D, Akpo LE. 2011. Effect of water regimes and pre-sowing treatments on seeds germination of different provenances of Jatropha curcas L. in Senegal. Int. J. Sci. Adv Tech., 1(9): 151-156. DOI: http://www.ijsat.com 
Orwa C. 2009. Agroforestry database: a tree reference and selection guide, version 4.0: Jatropha curcas, http://www.worldagroforestry.org/treed b/AFTPDFS/Jatropha_curcas.pdf, (21/05/2014).

Pompelli MF, Ferreira DTRG, Cavalcante PPGS, Salvador TL, Hsie BS, Endres L. 2010. Environmental influence on the physico-chemical and physiological properties of Jatropha curcas L. seeds. Aust. J. Bot., 58(6): 421-427. DOI: 10.1071/BT10102

Rajaona AM, Brueck H, Asch F. 2011. Effect of pruning history on growth and dry mass partitioning of Jatropha on a plantation site in Madagascar. Biomass \& Bioenergy, 35: 4892-4900.

Reinhard K. Henning, Tianasoa Ramorafeno, 2005. Le manuel de Jatropha (un guide pour l'exploitation intégrée de la plante de Jatropha à Madagascar). Green Island Association. Madagascar, pp118 www.Jatropha.org

Reubens B, Achten WMJ, Maes WH, Danjon F, Aerts R, Poesen J, Muys B, 2011. More than biofuel? Jatropha curcas root system symmetry and potential for soil erosion control. J. Arid. Environ., 75(2011): 201-205. DOI: 10.1016/ j.jaridenv.2010.09.011

Sanou F. 2010. Productivité de Jatropha curcas $L$. et impact de la plante sur les propriétés chimiques du sol : cas de BAGRE (Centre Est du BURKINA FASO). Diplôme d'ingénieur du développement rural (option: Eaux et Forêts). Université Polytechnique de BOBO-DIOULASSO : Institut du Développement Rural. 72 pages.

Tiwari AK, Kumar A, Raheman H. 2007. Biodiesel production from jatropha oil (Jatropha curcas) with high free fatty acids: An optimized process. Biomass \& Bioenergy, 31(8): 569-575. DOI: 10.1016/j.biombioe.2007.03.003

Trabucco A, Achten W, Bowe C, Aerts R, Van Orshoven J, Norgrove L, Muys B. 2010. Global mapping of Jatropha curcas yield based on response of fitness to present and future climate. GCB Bioenergy., 2(3): 139 - 151. DOI: $10.1111 / \mathrm{j} .1757-1707.2010$. 01049.x

Wen Y, Min T, Dongjing S, Hongguang Z, Jie W, Fong C, Lin T. 2012. Influence of climatic factors and soil types on seed weight and oil content of Jatropha curcas in Guangxi, China. Procedia Environ. Sci., 12(2012) 439-444. DOI: 10.1016/j.proenv.2012.01301 